\title{
ASSOCIAÇÃO ENTRE GRADIENTE DE PRESSÃO PORTAL E ASCITE EM PACIENTES COM CIRROSE
}

\author{
Sirlei DITTRICH ${ }^{1}$, Angelo Alves de MATTOS ${ }^{2}$, Ângelo Zambam de MATTOS ${ }^{2}$, \\ Alexandro Vaesken ALVES $^{2}$ and Fernanda Branco de ARAÚJO²
}

\begin{abstract}
RESUMO - Contexto - A hipertensão portal exerce papel importante na patogênese da ascite. Objetivo - Avaliar o gradiente de pressão venosa hepática e a presença de ascite em pacientes com cirrose. Métodos - Foram estudados 83 pacientes com cirrose. Todos os doentes realizaram estudo ecográfico para a identificação de ascite e foram submetidos a estudo hemodinâmico hepático para determinação do gradiente de pressão venosa hepática. Resultados - Na população avaliada, observou-se ascite em 70 doentes $(84,3 \%)$, sendo que a média geral do gradiente de pressão venosa hepática foi de 15,26 $\pm 6,46 \mathrm{~mm} \mathrm{Hg}$. Não houve diferença estatisticamente significante $(P$ $=0,061)$ quando avaliadas as médias do gradiente de pressão venosa hepática nos pacientes com $(14,70 \pm 6,43 \mathrm{~mm} \mathrm{Hg})$ e sem ascite $(18,64 \pm 5,78 \mathrm{~mm} \mathrm{Hg})$. Ao ser utilizado nível de corte de $8 \mathrm{~mm} \mathrm{Hg}$, para se avaliar o risco do desenvolvimento de ascite, observou-se que nos pacientes que apresentaram o gradiente de pressão venosa hepática superior a $8 \mathrm{~mm} \mathrm{Hg}$, o risco relativo do desenvolvimento de ascite foi de $0,876(0,74-1,03), P=0,446$. Conclusão - Nível pressórico de $8 \mathrm{~mm} \mathrm{Hg}$, determinado pelo gradiente de pressão venosa hepática, não define a presença ou ausência de ascite no paciente cirrótico e, tendo em vista a similitude das médias de pressão dos pacientes com e sem derrame peritonial, não se pode definir um ponto de corte para o surgimento de tal complicação.
\end{abstract}

DESCRITORES - Pressão na veia porta. Hipertensão portal. Ascite. Cirrose hepática.

\section{INTRODUÇÃO}

A hipertensão portal (HP) é uma síndrome clínica caracterizada por aumento patológico na pressão venosa portal e pela formação de colaterais portossistêmicas, através das quais ocorre desvio do fluxo sanguíneo portal para a circulação sistêmica ${ }^{(6)}$. A causa da HP é vasta, porém, em países desenvolvidos, a cirrose é a principal causa, sendo responsável por mais de $90 \%$ dos $\operatorname{casos}^{(6)}$. A principal complicação da HP é a hemorragia digestiva por ruptura de varizes gastroesofágicas ${ }^{(2)}$, no entanto, exerce papel fundamental na patogênese da ascite.

A ascite é a complicação mais comum da cirrose ${ }^{(3)}$. Seu desenvolvimento ocorre em torno de $50 \%$ dos pacientes em 10 anos de acompanhamento, empobrecendo o prognóstico dos mesmos, uma vez que sua presença empresta mortalidade de $50 \%$ em 3 anos ${ }^{(3)}$.

A presença de ascite não se deve exclusivamente à HP, embora ela seja o principal fator, sem o qual não é iniciada toda a série de eventos patogênicos envolvidos em seu desenvolvimento.

A avaliação da presença de HP pode ser feita a partir de dados clínicos, laboratoriais, procedimentos endoscópicos e imagéticos, mas a medida da pressão portalé o procedimento mais apropriado para avaliar sua real elevação ${ }^{(5,8,11,12,13,15,23)}$.

Tem-se preferido a determinação da pressão portal através de método indireto. Nesse caso, utilizam-se pontos de referência internos, como a pressão da veia cava inferior ou da veia hepática, determinando-se, então, um gradiente de pressão venosa portal ${ }^{(25)}$. O método indireto de medida utilizado que reflete de forma fidedigna a pressão venosa portal é a pressão venosa hepática ocluída, pois ocluíndo-se um ramo da veia hepática, a pressão medida será a do espaço sinusoidal. Essa técnica foi desenvolvida por Myers e Taylor ${ }^{(25)}$ e tem sido a mais utilizada para tal. Nesse método, o ponto de referência é a pressão venosa hepática livre, medida na junção da veia hepática e/ ou na veia cava inferior. A medida da pressão venosa hepática ocluída e livre fornece gradiente entre essas duas pressões, o gradiente de pressão venosa hepática (GPVH), que é um método indireto, simples e seguro para a avaliação da pressão portal, que vem sendo utilizado há mais de $50 \operatorname{anos}^{(2,4,14,16,15,31)}$.

Em indivíduos normais, o gradiente entre a pressão venosa hepática ocluída e livre varia de 1 até 4 ou 5 $\mathrm{mm} \mathrm{Hg}$. Acima desses valores, considera-se existir HP.

\footnotetext{
Curso de Pós-graduação em Hepatologia, Universidade Federal de Ciências da Saúde de Porto Alegre e Serviço de Hemodinâmica da Irmandade Santa Casa de Misericórdia de Porto Alegre, RS.

${ }^{1}$ Serviço de Gastroenterologia Clínica e Cirúrgica, Irmandade Santa Casa de Misericórdia de Porto Alegre, RS.: ${ }^{2}$ Disciplina de Gastroenterologia, Universidade Federal de Ciências da Saúde, Porto Alegre, RS.

Correspondência: Dra. Sirlei Dittrich - Rua Artur Rocha, 920/401 - 90450-170 - Porto Alegre, RS. E-mail: sirleid@terra.com.br
} 
Alguns estudos ${ }^{(24,30)}$ correlacionam o GPVH com o surgimento de ascite em pacientes cirróticos, sendo utilizado como valor crítico discriminativo o de $8 \mathrm{~mm} \mathrm{Hg}$, abaixo do qual não se desenvolveria o derrame peritonial.

Este estudo teve como objetivo avaliar a medida da pressão portal, realizada através do GPVH e a presença de ascite em pacientes com cirrose.

\section{MATERIAL}

Em período de 5 anos consecutivos foram estudados 83 pacientes com hepatopatia crônica internados na enfermaria de Gastroenterologia e Hepatologia da Irmandade Santa Casa de Misericórdia de Porto Alegre, RS, através de estudo hemodinâmico hepático, realizado no Serviço de Hemodinâmica da mesma instituição. Todos realizaram o referido estudo com o intuito de avaliar o GPVH antes da introdução da terapia farmacológica na profilaxia do sangramento decorrente da ruptura de varizes gastroesofágicas. Estes pacientes iniciaram terapia farmacológica por terem história de sangramento prévio, por ruptura de varizes gastroesofágicas $(68,7 \%)$ ou por terem varizes de grosso calibre $(31,3 \%)$.

O diagnóstico de cirrose foi confirmado por dados clínicos, laboratoriais e ecográficos e/ou exame anatomopatológico, sendo que este foi realizado em $16,9 \%$ dos pacientes. O exame anatomopatológico não foi realizado em um número maior de pacientes por alteração severa da coagulação ou pela riqueza dos dados clínicos, laboratoriais e/ou ecográficos a sugerirem o diagnóstico de cirrose.

Para ser definida a causa viral da hepatopatia crônica foram realizados o anti-HCV e o HBsAg. A causa alcoólica foi definida quando houvesse ingestão maior de 80 gramas de etanol por dia, durante período igual ou superior a 5 $\operatorname{anos}^{(14,27,35)}$.

$\mathrm{Na}$ avaliação da função hepatocelular foi utilizada a classificação de Child-Pugh ${ }^{(29)}$.

\section{MÉTODOS}

Todos os pacientes realizaram estudo ecográfico para a identificação de ascite e foram submetidos a estudo hemodinâmico hepático para determinação do GPVH.

A cateterização da veia hepática foi obtida através de punção da veia femoral. A pressão venosa hepática foi medida através da colocação de um cateter radiopaco na veia hepática sob controle radioscópico. Conectado ao cateter estava um fisiógrafo, que registrava curva de pressão. A pressão venosa hepática ocluída foi mensurada com a ponta do cateter bloqueando um ramo da veia hepática. Confirmou-se a posição correta de oclusão da mesma através dos seguintes critérios, seguindo os preceitos sugeridos por Groszmann et al. ${ }^{(15)}: 1$. presença de curva de pressão estável; 2 . ausência de refluxo de material de contraste para a veia hepática, ou seja, era injetado contraste, que deveria progredir e opacificar o leito sinusoidal e não refluir; 3 . queda importante da pressão na retirada do cateter, ou seja, o cateter era tracionado e dever-se-ia registrar diminuição na curva de pressão. Em geral, as medidas da pressão venosa hepática ocluída em diferentes veias hepáticas, em pacientes com cirrose, são idênticas. Assim, o valor obtido inicialmente era confirmado pela oclusão de um segundo ramo da veia hepática pelo cateter. Se houvesse discrepância entre os dois valores, a pressão ocluída era mensurada novamente em uma terceira veia hepática. O terceiro valor, usualmente idêntico a um dos dois prévios, foi usado como valor final. A pressão venosa hepática livre foi mensurada na junção da veia hepática e da veia cava inferior ou na veia cava inferior ${ }^{(15)}$. O GPVH foi obtido através da diferença entre a pressão venosa ocluída e livre.

Os seguintes critérios foram observados como contraindicação à realização do estudo hemodinâmico: idade menor de 18 anos, gravidez, mau estado geral, encefalopatia portossistêmica, peritonite bacteriana espontânea, hemorragia digestiva aguda, insuficiência cardíaca grau IV, insuficiência respiratória grave, alterações hemodinâmicas graves, atividade de protrombina abaixo de $50 \%$, contagem de plaquetas abaixo de $50000 \mathrm{cel} / \mu \mathrm{L}$. Ressalte-se haver estudo recente que concluiu que a atividade anormal de protrombina não estava relacionada com aumento no risco de sangramento após angiografia ${ }^{(34)}$.

A não-canulação da veia hepática por problemas técnicos, também constituiu critério de exclusão.

Este projeto foi submetido a analise da Comissão de Ética da Irmandade Santa Casa de Misericórdia de Porto Alegre, sendo exigido de todos os pacientes consentimento informado para a realização do estudo.

$\mathrm{Na}$ avaliação estatística foi utilizado o teste exato de Fisher, sendo empregado o programa SPSS 8.0. Foi considerado erro alfa de $5 \%(P<0,05)$. As variáveis quantitativas foram apresentadas em forma de média e desvio-padrão e as variáveis categóricas em forma de frequência e percentagem.

\section{RESULTADOS}

Dos 83 pacientes, $59(71,1 \%)$ eram homens e $24(28,9 \%)$, mulheres. A idade variou de 26 a 75 anos, tendo média de 52,9 anos, com desvio-padrão de 10,1 anos. Em relação à cor, 71 pacientes $(85,5 \%)$ eram brancos e $12(14,5 \%)$, pretos.

Quanto à etiologia, em 25 pacientes $(30,1 \%)$, o álcool era o único fator envolvido na causa da hepatopatia; em 26 $(31,3 \%)$, o álcool estava associado aos vírus da hepatite B ou C; em $28(33,7 \%)$, a causa era associada à hepatite pelos vírus $\mathrm{B}$ ou $\mathrm{C}$; a cirrose foi considerada criptogênica em 4 pacientes $(4,8 \%)$.

Os pacientes estudados foram divididos em grupos conforme a classificação de Child-Pugh. Assim, 23 pacientes $(27,7 \%)$ eram Child A, 48 (57,8\%) eram Child B, 11 (13,3\%) eram Child $\mathrm{C}$ e em $1(1,2 \%)$ não se obteve este dado por extravio do registro.

Dos 83 pacientes, $57(68,7 \%)$ haviam apresentado sangramento prévio por ruptura de varizes e $26(31,3 \%)$ tinham varizes de grosso calibre, mas nunca haviam sangrado.

$\mathrm{Na}$ população de pacientes avaliados, observou-se ascite em 70 doentes $(84,3 \%)$. 
Avaliando o estudo hemodinâmico nos 83 pacientes hepatopatas crônicos, viu-se que a média geral do GPVH foi de $15,26 \pm 6,46 \mathrm{~mm} \mathrm{Hg}$.

Não houve diferença estatisticamente significante $(P=$ $0,061)$ quando avaliadas as médias do GPVH nos pacientes com $(14,70 \pm 6,43 \mathrm{~mm} \mathrm{Hg})$ e sem ascite $(18,64 \pm 5,78 \mathrm{~mm} \mathrm{Hg})$.

Foi utilizado nível de corte para se avaliar o risco do desenvolvimento de ascite, para tal, utilizou-se o valor de $8 \mathrm{~mm} \mathrm{Hg}$. Entre os pacientes que apresentaram o GPVH superior a $8 \mathrm{~mm} \mathrm{Hg}$, o risco relativo do desenvolvimento de ascite foi de $0,876(0,74-1,03), P=0,446$.

A fim de se calcular o valor preditivo positivo e negativo para a medida de $8 \mathrm{~mm} \mathrm{Hg}$ para a presença de ascite, os dados estão resumidos no seguinte:

\begin{tabular}{lcc}
\hline & Ascite & \\
\hline & Sim & Não \\
$\geq 8 \mathrm{~mm} \mathrm{Hg}$ & 59 & 12 \\
$<8 \mathrm{~mm} \mathrm{Hg}$ & 11 & 1 \\
TOTAL & 70 & 13 \\
\hline
\end{tabular}

O valor preditivo positivo calculado do GPVH de $8 \mathrm{~mm}$ $\mathrm{Hg}$ para presença de ascite foi de $83 \%$, enquanto que o valor preditivo negativo foi de $8 \%$.

\section{DISCUSSÃO}

A HP é definida como uma elevação do gradiente de pressão portal que exceda $5 \mathrm{~mm} \mathrm{Hg}^{(6)}$.

A medida dos níveis da pressão portal, através do GPVH, pode auxiliar no diagnóstico da $\mathrm{HP}^{(5,15,19)}$, na avaliação do risco de sangramento por ruptura de varizes gastroesofágicas ${ }^{(11}$, 14, 34, 35), na avaliação de eficácia do tratamento de sua maior complicação, o sangramento por varizes gastroesofágicas ${ }^{(1,17}$. ${ }^{33,34)}$, na decisão terapêutica em casos de ressecção hepática ${ }^{(10}$, ${ }^{20)}$ e na avaliação do prognóstico dos pacientes ${ }^{(1,2,22,34,35)}$.

A despeito do GPVH não ser realizado na avaliação diagnóstica da ascite, observação feita é a de que os níveis do GPVH se correlacionam com o surgimento de ascite ${ }^{(24,30)}$.

Assim, Morali et al. ${ }^{(24)}$, ao avaliarem 522 cirróticos com e sem ascite, observaram que nenhum dos pacientes com ascite apresentava pressão sinusoidal, medida através de avaliação hemodinâmica por via transjugular, menor de $8 \mathrm{~mm} \mathrm{Hg}$.

$\operatorname{Rector}^{(30)}$, por sua vez, avaliou a pressão portal em 124 pacientes com doença hepática crônica e também observou que a mais baixa medida de pressão portal verificada em pacientes com ascite foi de $8 \mathrm{~mm} \mathrm{Hg}$.

Dois estudos ${ }^{(1,35)}$ sobre prevenção do sangramento por varizes de esôfago, observaram que o risco de desenvolvimento de ascite foi menor nos pacientes respondedores ao uso de beta-bloqueadores. Sugere-se, assim, que, ao se baixarem os níveis da pressão do sistema porta, diminui-se a probabilidade dos pacientes desenvolver derrame peritonial.

No presente estudo, a alta percentagem de pacientes com cirrose e ascite é explicada pelo fato dos mesmos estarem internados em serviço de referência em hepatologia, muitos dos quais em avaliação para transplante hepático. Quando foram comparadas as médias do GPVH nos 83 pacientes com e sem ascite, não houve diferença significante entre tais valores. Além disso, foram observados 11 pacientes com ascite e GPVH inferior a $8 \mathrm{~mm} \mathrm{Hg}$ (valor preditivo negativo $=8 \%$ ), contrariando os estudos anteriormente citados ${ }^{(24,30)}$.

A medida da pressão portal pode oferecer resultados heterogêneos, influenciados por múltiplos fatores. Assim, quando avaliados pacientes com cirrose decorrente do uso de álcool, parece que a determinação do GPVH é mais fidedigna do que quando avaliados pacientes em que a hepatopatia é decorrente de vírus, quando a pressão estaria subestimada ${ }^{(7,}$ 28). Por outro lado, mesmo em população de pacientes em que o álcool está implicado na gênese da hepatopatia, na dependência de sua supressão, pode haver diminuição dos níveis pressóricos ${ }^{(2,17,21,36)}$. Estudos experimentais têm demonstrado que a administração de etanol aumenta o fluxo sanguíneo esplâncnico em ratos normais e com HP, assim como em pacientes cirróticos. Além disso, tem sido demonstrado que o etanol tem efeito vasoconstritor na microcirculação hepática. A combinação do aumento do fluxo portal e da resistência vascular hepática leva a aumento da pressão portal ${ }^{(9,21,32)}$. Logo, a abstinência resulta em queda do GPVH. Ressalte-se que a pressão portal também sofre oscilações, dependendo do período, na história natural da hepatopatia, em que é realizada, sem que haja justificativa para o fato ${ }^{(17)}$.

A despeito desses fatores, os achados do presente estudo sugerem, além da HP, a participação mais efetiva de outros mecanismos na fisiopatologia do derrame peritonial desta população de pacientes. A HP e níveis baixos de albumina sérica contribuem para o aparecimento de ascite, porém há outros fatores envolvidos, concorrendo para a precipitação das alterações hemodinâmicas, em nível sistêmico e em nível renal, decorrentes da liberação de substâncias vasoativas, e culminando com retenção de sódio e de água ${ }^{(26)}$.

\section{CONCLUSÃO}

Concluí-se, no presente estudo, que nível pressórico de $8 \mathrm{~mm} \mathrm{Hg}$, determinado pelo GPVH, não revela de forma definitiva a presença ou ausência de ascite no paciente cirrótico e que, tendo em vista a similitude das médias de pressão dos pacientes com e sem derrame peritonial e o baixo valor preditivo negativo deste valor, não se pode definir um ponto de corte para o surgimento de tal complicação. 
Dittrich S, Mattos AA, Mattos AZ, Alves AV, Araújo FB. Relationship between portal pressure gradient and ascites in cirrhotic patients. Arq Gastroenterol. 2010;47(2):174-7.

ABSTRACT - Context - Portal hypertension plays an important role in the pathogenesis of ascites. Objectives - To evaluate the hepatic venous pressure gradient and the presence of ascites in cirrhotic patients. Methods - Eighty-three patients with cirrhosis were evaluated. All of the patients were submitted to ultrasonography to identify ascites and to a hepatic hemodynamic investigation to determine the hepatic venous pressure gradient. Results - In the population evaluated, ascites was observed in 70 patients $(84.3 \%)$, and the mean hepatic venous pressure gradient was $15.26 \pm 6.46$ $\mathrm{mm} \mathrm{Hg}$. There was no statistically significant difference $(P=0.061)$ between the means of hepatic venous pressure gradient in patients with $(14.70$ $\pm 6.43 \mathrm{~mm} \mathrm{Hg}$ ) and without ascites $(18.64 \pm 5.78 \mathrm{~mm} \mathrm{Hg})$. When using a cut-off point of $8 \mathrm{~mm} \mathrm{Hg}$ in order to assess the risk of developing ascites, patients with hepatic venous pressure gradient above $8 \mathrm{~mm} \mathrm{Hg}$ were found to have a relative risk of $0.876(\mathrm{CI}=0.74-1.03),(P=0.446)$ of progressing to ascites. Conclusions - The pressure level of $8 \mathrm{~mm} \mathrm{Hg}$, as determined by the hepatic venous pressure gradient, does not define the presence or absence of ascites in the cirrhotic patient, and in view of the similarity between mean pressures in patients with or without peritoneal effusion, it is impossible to define a cut-off point for the emergence of such complication.

HEADINGS - Portal pressure. Hypertension, portal. Ascites. Liver cirrhosis.

\section{REFERENCES}

1. Abraldes JG, Tarantino I, Turnes J, Garcia-Pagan JC, Rodes J, Bosch J. Hemodynamic response to pharmacological treatment of portal hypertension and long-term prognosis of cirrhosis. Hepatology. 2003;37:902-8.

2. Armonis A, Patch D, Burroughs A. Hepatic venous pressure measurement: an old test as a new prognostic marker in cirrhosis? Hepatology. 2007;25:245-8.

3. Arroyo V, Colmenero J. Ascite and hepatorrenal syndrome in cirrhosis: pathophysiological basis of therapy and current management. J Hepatol. 2003;38:s69-s89.

4. Bean WB, Paul WD, Franklin M. Preliminary studies on an indirect method for determining portal pressure. J Clin Invest. 1949;28:769-70.

5. Bosch J, Navasa M, Kravetz D, Pizcueta MP, Garcia-Pagán JC, De Lacy AM, Rodés J. Diagnosis and evaluation of portal hypertension. Z Gastroenterol. 1988;26:8-14.

6. Bosch J, Navasa M, Garcia-Pagan JC, DeLacy AM, Rodes J. Portal hypertension. Med Clin North Am. 1989;73:931-53.

7. Boyer TD, Triger DR, Horisawa M, Redeker AG, Reynolds TB. Direct transhepatic measurement of portal vein pressure using a thin needle. Comparison with wedged hepatic vein pressure. Gastroenterology. 1977;72:584-9

8. Brazzini A, Hunter DW, Darcy MD, Smith TP, Cragg AH, Castaneda-Zuniga WR, Amplatz K. Safe splenoportography. Radiology. 1987;162:607-9.

9. Bredfeldt JE, Riley EM, Groszmann RL. Compensatory mechanisms in response to an elevated hepatic oxygen consumption in chronically ethanol-fed rats. Am J Physol. 1985;248:g507-g11.

10. Bruix J, Castells A, Bosch J, Feu F, Fuster J, Garcia-Pagan JC, Visa J, Bru C, Rodes J. Surgical resection of hepatocellular carcinoma in cirrhotic patients: prognostic value of preoperative portal pressure. Gastroenterology. 1996;111:1018-22.

11. D'amico G, Pagliaro L, Bosch J. The treatment of portal hypertension: a metaanalytic review. Hepatology. 1995;22:332-48.

12. Debaene B, Goldfarb G, Braillon A, Jolis P, Lebrec D. Effects of ketamine, halothane, enflurane and isoflurane on systemic and splanchnic hemodynamics in normovolemic and hypovolemic cirrhotic rats. Anesthesiology. 1990;73:118-24.

13. Fenyves D, Pomier-Layrargues G, Willems B, Cote J. Intrahepatic pressure measurement: not an accurate reflection of portal vein pressure. Hepatology. 1988;8:211-6.

14. Garcia-Tsao G, Groszmann RJ, Fisher RL, Conn HO, Atterbury CE, Glickman M. Portal pressure, presence of gastroesophageal varices and variceal bleeding. Hepatology. 1985;5:419-24.

15. Groszmann RJ, Glickman M, Blei AT, Storer E, Conn HO. Wedged and free hepatic vein venous pressure measured with baloon catheter. Gastroenterology. 1979;76:253-8.

16. Groszmann RJ, Atterbury LE. The pathophysiology of portal hypertension: a basis for classification. Semin Liver Dis. 1982;2:217-86.

17. Groszmann RJ, Bosch J, Grace ND, Conn HO, Garcia-Tsao G, Navasa M, Alberts J, Rodes J, Fischer R, Bermann M. Hemodynamic events in a prospective randomized trial of propranolol versus placebo in the prevention of a first variceal hemorrhage. Gastroenterology. 1990;99:1401-7.

18. Groszmann RJ, Garcia-Tsao G. Endoscopic variceal banding vs. pharmacological therapy for the prevention of recurrent variceal hemorrhage: what makes the difference? Gastroenterology. 2002;123:1388-91.

19. Lebrec D, Benhamou JP. Noncirrhotic intrahepatic portal hypertension. Sem Liver Dis. 1986;6:332-40.
20. Llovet JM, Fuster J, Bruix J. Intention-to-treat analysis of surgical treatment for early hepatocellular carcinoma: resection versus transplantation. Hepatology 1999;30:1434-40.

21. Luca A, Garcia-Pagan JC, Bosch J, Feu F, Caballeria J, Groszmann RJ, Rodes J. Effects of ethanol consumption on hepatic hemodynamics in patients with alcoholic cirrhosis. Gastroenterology. 1997;112:1284-9.

22. Merkel C, Bolognesi M, Bellon S, Zuin R, Noventa F, Finucci G, Sacerdoti D, Angeli P, Gatta A. Prognostic usefulness of hepatic vein catheterization in patients with cirrhosis and esophageal varices. Gastroenterology. 1992;102: 973-9.

23. Moore GE, Bridenbawch RB. Portal venography. Surgery. 1950;28:827-31.

24. Morali GA, Sniderman KW, Deitel KM, Tobe S, Witt-Sullivan H, Simon M, Heathcote J, Blendis LM. Is sinusoidal portal hypertension a necessary factor for the development of hepatic ascites? J Hepatol. 1992;16:249-50.

25. Myers JD, Taylor WJ. An estimation of portal venous pressure by occlusive catheterization of an hepatic venule. J Clin Invest. 1951;30:662-3.

26. Navasa M, Arroyo V. Ascites and spontaneous bacterial peritonitis. In: Schiff ER, Sorrell MF, Maddrey WC, editors. Schiff's disease of the liver Philadelphia: Lippincott; 2003. p.559-94.

27. Perello A, Escorsell A, Bru C, Gilabert R, Moitinho E, Garcia-Pagan JC, Bosch J. Wedged hepatic venous pressure adequately reflects portal pressure in hepatitis C virus-related cirrhosis. Hepatology. 1999;30:1393-7.

28. Pomier-Layrargues G, Kusielewicz D, Willems B, Villeneuve JP, Marleau D, Cote J, Huet PM. Presinusoidal portal hypertension in non-alcoholic cirrhosis. Hepatology. 1985;5:415-8.

29. Pugh RN, Murray-Lyon IM, Dawson JL. Transection of the oesophagus of bleeding oesophageal varices. Br J Surg. 1973;60:646-9.

30. Rector WG. Portal hypertension: a permissive factor only in the development of ascites and variceal bleeding. Liver. 1986;6:221-6.

31. Taylor WJ, Myers JD. Occlusive hepatic venous catheterization in the study of the normal liver, cirrhosis of the liver and noncirrhotic portal hypertension. Circulation. 1956;13:368-80.

32. Verma-Ansil B, Carmichael FJ, Saldivia V, Varghese G, Orrego H. Effect of ethanol on splanchnic hemodynamics in awake and unrestrained rats with portal hypertension. Hepatology. 1989;6:946-52.

33. Viallet A, Marleau D, Huet M, Martin F, Farley A, Villeneuve JP, Lavoie P. Hemodynamic evaluation of patients with intrahepatic portal hypertension. Relationship between bleeding varices and the portohepatic gradient. Gastroenterology. 1975;69:1297-300.

34. Villanueva C, Minana J, Ortiz J, Gallego A, Soriano G, Torras X, Sainz S, Boadas J, Cusso X, Guarner C, Balanzo J. Endoscopic compared with combined treatment with nadolol and isosorbide mononitrate to prevent recurrent variceal bleeding. New Engl J Med. 2001;345:647-55.

35. Villanueva C, Lopez-Balaguer JM, Aracil C, Kolle L, Gonzalez B, Minana J, Soriano G, Guarner C, Balanzo J. Maintenance of hemodynamic response to treatment for portal hypertension and influence on complications of cirrhosis. $\mathrm{J}$ Hepatol. 2004;40:757-65.

36. Vorobioff J, Groszmann RJ, Picabea E, Gamen M, Villavicencio R, Bordato J, Morel I, Audano M, Tanno H, Lerner E, Passamonti M. Prognostic value of hepatic venous pressure gradient measurements in alcoholic cirrhosis: a 10 years prospective study. Gastroenterology. 1996;111:701-9. 